\title{
Analysis of Antennas by FDTD Method Using Parallel Processing with MPI
}

\author{
Josivaldo de S. Araújo, Ronaldo O. Santos, Carlos L. da S. S. Sobrinho, Johnny M. Rocha, Luiz A. Guedes and \\ Regiane Y. Kawasaki \\ Federal University of Pará (UFPA), DEEC, CEP: 66075-900, Belém, PA.
}

\begin{abstract}
As far as we are concerned, the implementation of the Finite Differences in the Time Domain (FDTD) method requires, for the solutions of several practical problems in electromagnetism, a long process time and a large amount of memory, what makes it impracticable in various cases, principally when the serial process is used. The current work deals with the conception of a Beowulf cluster and it aims to implement the FDTD method using parallel process for the study of antennas. The obtained system efficiency is then tested in the analysis of two antennas, i. e., a horn antenna and a monopole antenna, what is done by comparing the time spent in the parallel and serial processing.
\end{abstract}

Keywords: FDTD method, cluster, horn antenna, monopole antenna and parallel implementation.

\section{INTRODUCTION.}

The Finite Differences in the Time Domain (FDTD) method was introduced by Yee, in 1966 [1], and represents a simple and efficient form of solving Maxwell's equations when written in the differential-time domain form. In Yee's proposal, the components of electric and magnetic fields are intercalated in space and time, in such a way that there is reciprocity among them.

The FDTD method was first used to analyze the electromagnetic waves scattering by objects. Later, after the development of techniques to include sources in the computational domain, the method was used to analyze the radiation characteristics of structures [2]. Since the first use of the FDTD method in the analysis of simple, cylindrical monopole and horn antennas [3], until now, there has been a great evolution of numerical techniques and the appearance of new generation of computers that allowed that the FDTD method could be used in the analysis of complex structures. Among the numerical techniques, one can mention: the Berenger's PML (Perfectly Matched layer), proposed in 1994 [4] and the FDTD algorithm to general nonorthogonal grids [5]. In relation to the computational development, one can mention the parallel computation, which is the main focus of this article.

In spite of its credibility and precision, the FDTD method has its limits. One of them is the great amount of memory required, and the other one is the long processing time.

J. S. Araújo, isaraujo@interconect.com.br, R. O. Santos, ronaldo@ufpa.br, , C. L. S. S. Sobrinho, leonidas@ufpa.br.

This work was supported by $\mathrm{CNPq}$ and CAPES.
A solution for these problems would be the use of more powerful computers, in particular, several processors working at a parallel structure. However, the access to these supercomputers is not simple yet, due to the high cost and to very specific purposes. Another alternative, more financially accessible, would be the use of a technique, developed in the late 80 's, that is the cluster architecture of PC's [6].

The implementation of architecture of parallel computers, the known cluster, is developed through software and libraries of message passing, where communication among the interconnected computers is done via data communication network. The most used public domain libraries are the PVM (Parallel Virtual Machine) and the MPI (Message Passing Interface). These libraries are defined for the programming languages, $\mathrm{C} / \mathrm{C}++$ and FORTRAN.

As a part of this project the DEEC/UFPA Laboratory of Numerical Analysis in Electromagnetics developed two clusters: The first one, named LANE1, is constituted of three machines and the second, named AMAZÔNIA-LANE2, is constituted of eight machines.

In this work, one is using a LAM/MPI library along with the FDTD method to make a precise analysis of two kinds of antennas: monopole antenna and horn antenna; and also to show the efficiency of the parallel computation in face to the sequential one. This efficiency will be shown through speedup (the parallel processing in relation to the sequential processing), obtained in the analysis of the antennas.

\section{MODELING OF THE ANTENNAS BY FDTD METHOD.}

In the present work, two antennas are analyzed: an E-plane sectoral horn antenna, that will be analyzed through the cluster LANE1 and; a cylindrical monopole antenna, over a PEC ground plane, will be analyzed through the cluster AMAZÔNIA. Each considered antenna was analyzed by using the serial and parallel processing. In the two ways of calculations, the space and time discretization were kept the same.

\section{A The E-plane sectorial horn antenna.}

The horn antenna is one of the simplest and probably the most widely used microwave antennas. That is because it presents a large bandwidth and it is easy to be constructed, ease of excitation, versatility, large gain, among other advantages [19]. In this way, the E-plane sectorial horn 
antenna may be used as a feed for reflectors and lenses, as shown in Fig.1, or radiating directly.

In Fig.2, it is shown the transversal section of the twodimensional model for the E-plane sectorial horn antenna with aperture of $14^{\circ}$. In this figure, all the parameters are normalized by the wavelength.

The horn antenna was chosen according to the optimum conditions, i.e., $B=\left(2 \lambda R_{2}\right)^{1 / 2}$ with $R_{2}=8 \lambda$ [7], and the waveguide with dimensions: $b=\lambda / 4$ and $l=2 \lambda$.

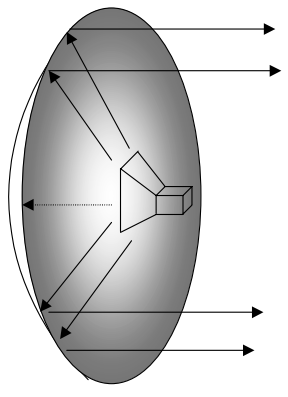

Fig.1. Parabolic Reflector fed by a horn antenna.

In the analysis made, only the TE mode is considered so that the problem is reduced in the following components $\left(E_{x}, E_{y}\right)$ for the electric field and $\left(H_{z}\right)$ for the magnectic field, which are expressed in the discretized form by:

$$
\begin{gathered}
H_{z}^{n+0.5}(i+0.5, j+0.5)=H_{z}^{n-0.5}(i+0.5, j+0.5)-\frac{\Delta t}{\mu_{0} \Delta x}\left[E_{y}^{n}(i+1, j+0.5)\right. \\
\left.-E_{y}^{n}(i, j+0.5)\right]-\frac{\Delta t}{\mu_{0} \Delta y}\left[E_{x}^{n}(i+0.5, j+1)\right. \\
\left.-E_{x}^{n}(i+0.5, j)\right], \\
E_{x}^{n+1}(i+0.5, j)=E_{x}^{n}(i+0.5, j)+\frac{\Delta t}{\varepsilon_{0} \Delta y} \\
\cdot\left[H_{z}^{n+0.5}(i+0.5, j+0.5)-H_{z}^{n+0.5}(i+0.5, j-0.5)\right] \\
E_{y}^{n+1}(i, j+0.5)=E_{y}^{n}(i, j+0.5)-\frac{\Delta t}{\varepsilon_{0} \Delta x} \\
\quad\left[H_{z}^{n+0.5}(i+0.5, j+0.5)-H_{z}^{n+0.5}(i-0.5, j+0.5)\right] .
\end{gathered}
$$

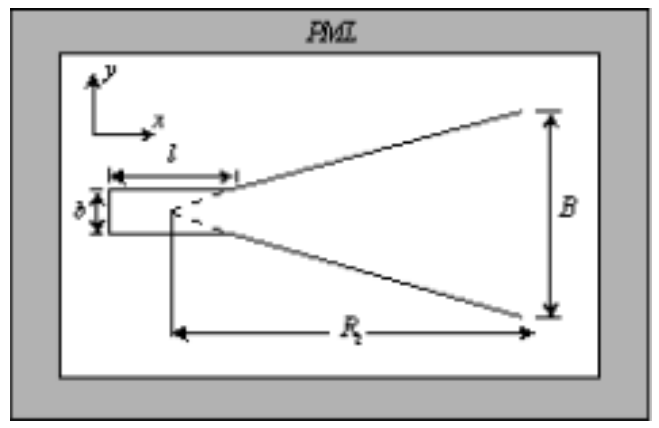

Fig.2. The E-plane Sectorial horn antenna with aperture of $14^{\circ}$.
In the discretization of the region of interest, one used a two-dimensional uniform mesh of $310 \times 248$, with cells dimensioned in space as $\lambda / 20$ per $\lambda / 20$ and in the time as $\Delta t \leq \Delta x / 2 c$ obeying the stability criterion suggested by Courant et al. [8].

As excitation function was used the sine modulated Gaussian pulse, expressed by:

$$
f(t)=1.484 \exp \left\{-\left[\left(t-3 \tau_{0}\right) / \tau_{0}\right]^{2}\right\} \cdot \sin \left(2 \pi f_{c} t\right),
$$

where

$\tau_{o}=\left[\pi\left(f_{h}-f_{c}\right)\right]^{-1}, f_{h}=15 \mathrm{GHz}, f_{c}=10 \mathrm{GHz}$, with $t=n . \Delta t$, $\Delta t=\lambda /(40 . c)$ and $\mathrm{c}=2.99792458 \mathrm{D}+8 \mathrm{~m} / \mathrm{s}$. Being, this source, positioned at $\lambda / 4$ from the back part of the guide (Fig.1).

With the aim of truncating the FDTD mesh, it was chosen to be used the uniaxial anisotropic PML technique developed by Gedney [9], because this technique has shown to be more efficient, under the computational aspect, than the PML of Berenger [4].

\section{B Cylindrical monopole over a PEC ground plane.}

The monopole antennas are widely used in wireless mobile communication [1]. Because of their characteristic of broadband and simple building, it is maybe the most common antenna for portable equipment, such as cellular telephones, cordless telephones, automobiles, trains, etc.

The cylindrical monopole antenna shown in Fig.3 represents a two-dimensional electromagnetic problem, because both the antenna and its source of feed are rotationally symmetric. The electromagnetic fields are independent of the angular coordinate $(\phi)$, and Maxwell's equations may be expressed as two independent groups: One that involves only the components $E_{r}, E_{z}, H_{\phi}$, TM mode or transverse magnetic mode; and other that involves only the components $E_{\phi}, H_{r}, H_{z}$, TE mode or transverse electric mode. Since the excitation of the antenna is made through a coaxial line, only the TM modes (with rotational symmetry) are excited. This way, this mode is used in our analysis. Maxwell's equations, for the TM mode in the discrete form and written in the cylindrical coordinate system, are given by:

$$
\begin{aligned}
H_{\phi}^{n+0.5}(i, j)= & H_{\phi}^{n-0.5}(i, j)+\frac{\Delta t}{\mu_{0} \Delta r}\left[E_{z}^{n}(i+0.5, j)\right. \\
- & \left.E_{z}^{n}(i-0.5, j)\right]-\frac{\Delta t}{\mu_{0} \Delta z}\left[E_{r}^{n}(i, j+0.5)\right. \\
- & \left.E_{r}^{n}(i, j-0.5)\right], \\
E_{r}^{n+1}(i, j-0.5)= & E_{r}^{n}(i, j-0.5)-\frac{\Delta t}{\varepsilon_{0} \Delta z} \\
& \cdot\left[H_{\phi}^{n+0.5}(i, j)-H_{\phi}^{n+0.5}(i, j-1)\right], \\
E_{z}^{n+1}(i+0.5, j)= & E_{z}^{n}(i+0.5, j)-\frac{\Delta t}{\varepsilon_{0} \Delta r} \frac{1}{r_{i+0.5}} \\
& \cdot\left[r_{i+1} H_{\phi}^{n+0.5}(i+1, j)-r_{i} H_{\phi}^{n+0.5}(i, j)\right] .
\end{aligned}
$$


The parameters that discribe the monopole antenna are: the height $h$ and the radii $a$ and $b$ from the coaxial line conductors. The antenna is characterized by the time $\tau_{a}=h / c$, that is the time needed for the light to go through the length of the antenna. In the region of analysis, one used a twodimensional uniform mesh of $600 \times 600$, with $\Delta r=\Delta z=h / 250$ and $\Delta t=h /(500 . c)$.

The cylindrical monopole was excited by a Gaussian pulse of $1 \mathrm{~V}$. The point of excitation was the transversal section B-B' located in the line of feed (Fig.2). In this transversal section, the incident electric field is given by:

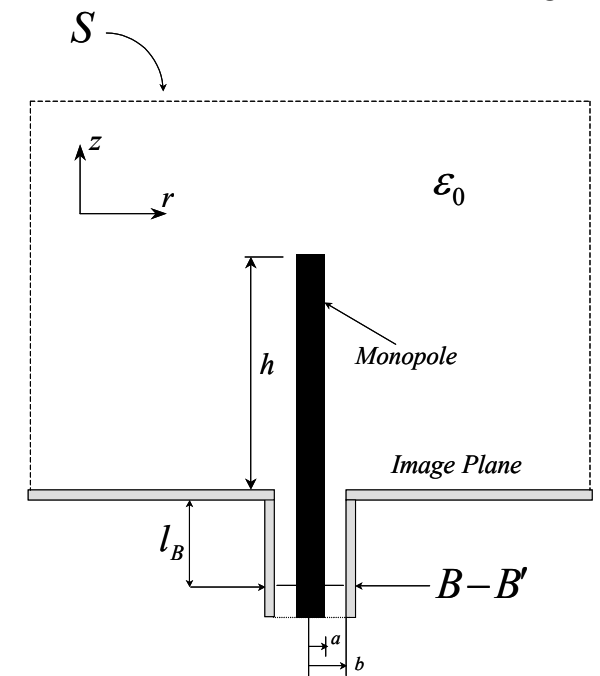

Fig.3. Geometry of the cylindrical monopole antenna fed through an image plane, by a coaxial transmission line.

$$
E^{i}(t)=\frac{V^{i}(t)}{\ln (b / a) r} \hat{r},
$$

where

$$
V^{i}(t)=V_{0} \exp \left(-t^{2} / 2 \tau_{p}^{2}\right)
$$

The B-B' plane taken as reference in the feed line was chosen so that the evanescent modes, present in the transition coaxial line - monopole antenna, are worthless, typically $l_{B}=3(b-a)[3]$.

In order to truncate the computational domain, one used as absorbing boundary condition (ABC), Mur 1st order [10], which is applied over the dashed surface $S$ (Fig.3).

\section{III.PARALLEL IMPLEMENTATION OF THE FDTD CODE.}

The main idea of the parallel implementation of the algorithm FDTD is based in the division of the domain of analysis in subdomains. In this technique, known as Data Decomposition or Domain Decomposition [11], the data of the problems are portioned among the different processors, considering that each processor executes basically the same program (source code), but on different data. This is a typical implementation of the SPMD model [13] (Single Program Multiple Data).

The parallel FDTD program is executed on an architecture of parallel machines of Cluster of PC's(COPs), where each machine has its own memory and local processor, what leads to the necessity of using a library that allows the exchange of messages among active processes in different machines. The library chosen to make the exchange of messages was the LAM-MPI, developed at the University of Ohio [12].

The distribution of data is manually made, i.e., it is the programmer that defines, through sending/receiving message functions, the communication among the surrounding processors in order to have a continuity in the updating of the components of field located on the interfaces of the domains.

For the horn antenna described before and considering the cluster with three machines, the domain of analysis was divided in equal subdomains along with the x-direction (Fig.4 (a)), resulting in matrixes of equal dimensions. Each matrix was stored and processed by only one processor, and each processor calculates the components of the electric and magnetic fields in the region of its domain. For example, for machine 1, we have $\left(H z_{1}, E x_{1}, E y_{1}\right)$; for machine 2, we have $\left(H z_{2}, E x_{2}, E y_{2}\right)$ and for machine 3, we have $\left(H z_{3}, E x_{3}, E y_{3}\right)$. Fig.4 (b) shows how the use of the components of the electric and magnetic fields are made, for the horn antenna, on the interfaces of the domains of each machine. Then, to calculate the components of the magnetic field in the instant $(n+0.5)$, for any cell of the subdomain I, in the border with the subdomain II, Eq.(1.a) is rewritten in (6)

$$
\begin{aligned}
H_{z l}^{n+0.5}(i+0.5, j+0.5)= & H_{z l}^{n-0.5}(i+0.5, j+0.5)-\frac{\Delta t}{\mu_{0} \Delta x}\left[E_{y 2}^{n}(i+1, j+0.5)\right. \\
& \left.-E_{y l}^{n}(i, j+0.5)\right]-\frac{\Delta t}{\mu_{0} \Delta y}\left[E_{x l}^{n}(i+0.5, j+1)\right. \\
& \left.-E_{x l}^{n}(i+0.5, j)\right]
\end{aligned}
$$

For this case, it is necessary to have the knowledge of the component of the electric field $E y_{2}$ (see Eq.(6) and Fig.4(b)), which was calculated by machine 2 , in the instant $n$, and sent by this machine to machine I in the same instant $n$.

Similar procedure is made for the use of the component $E y_{2}$ of the electric field for the instant $(n+1)$, considering any cell of the subdomain II, in the border with the subdomain I. In this case, the Eq. (1.c) is rewritten in (7), from where it is observed that it is necessary to have the knowledge of the component of the magnetic field $\mathrm{Hz}_{1}$ (see Eq.(7) and Fig.4(b)), which was calculated by machine 1 (Eq.(6)) in the earlier step and sent by this machine to machine 2 in the same instant $(n+0.5)$.

$$
\begin{aligned}
& E_{y 2}^{n+1}(i, j+0.5)=E_{y 2}^{n}(i, j+0.5)-\frac{\Delta t}{\varepsilon_{0} \Delta x} \\
& \cdot\left[H_{z 2}^{n+0.5}(i+0.5, j+0.5)-H_{z 1}^{n+0.5}(i-0.5, j+0.5)\right] .
\end{aligned}
$$

In the analysis of the cylindrical monopole antenna, considering the cluster with eight machines, the division of the region of interest was made along the r-direction (radial direction). Thus, the component $E_{z}^{n+1}$ of the electric field, for the subdomain I and on the border with the subdomain II, has dependence of the component $H_{\phi 2}$ of the magnetic field, as it can be noticed in the equation below: 


$$
\begin{aligned}
E_{z 1}^{n+1}(i+0.5, j)= & E_{z 1}^{n}(i+0.5, j)-\frac{\Delta t}{\varepsilon_{0} \Delta r} \frac{1}{r_{i+0.5}} \\
& \cdot\left[r_{i+1} H_{\phi 2}^{n+0.5}(i+1, j)-r_{i} H_{\phi 1}^{n+0.5}(i, j)\right],
\end{aligned}
$$

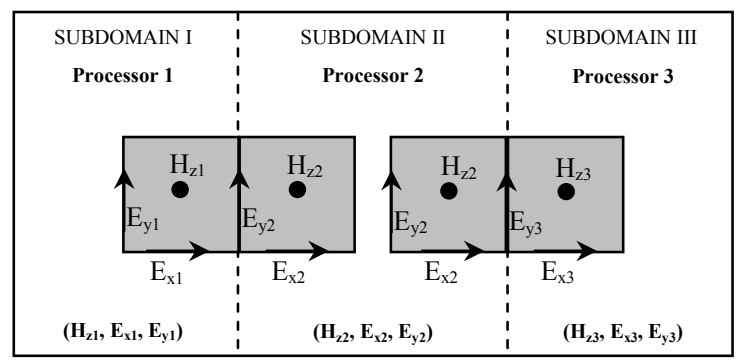

(a)

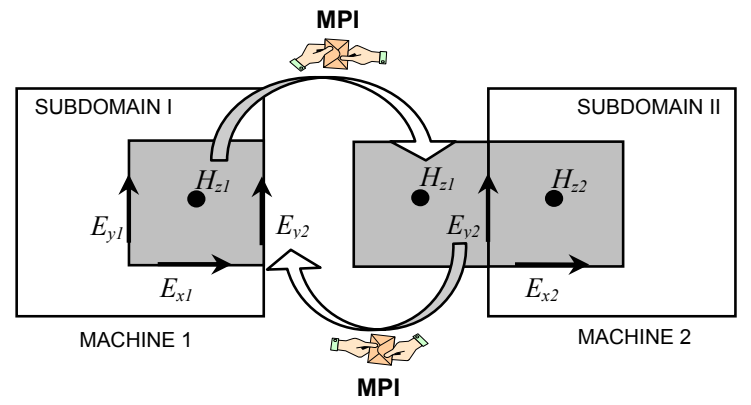

(b)

Fig.4. (a) Region of analysis divided in subdomains. (b) Updating of the components of field that are found at an interface between two regions.

This was obtained from (3.c). In Eq.(9), $H_{\phi 2}$ was obtained, in machine 2 in the instant $(n+0.5)$, and transferred to machine 1 in the same instant. The same procedure is applied to obtain the component $H_{\phi 2}^{n+0.5}$ of the magnetic field, for the subdomain II.

$$
\begin{aligned}
H_{\phi 2}^{n+0.5}(i, j)= & H_{\phi 2}^{n-0.5}(i, j)+\frac{\Delta t}{\mu_{0} \Delta r}\left[E_{z 2}^{n}(i+0.5, j)\right. \\
& -E_{z 1}^{n}(i-0.5, j)-\frac{\Delta t}{\mu_{0} \Delta z}\left[E_{r 2}^{n}(i, j+0.5)\right. \\
& -E_{r 2}^{n}(i, j-0.5),
\end{aligned}
$$

Thus, different processors work simultaneously executing a copy of the program, but about several data, i.e., the processor calculates all the components of the fields of its domain passing on only those located at the interface.

\section{IMPLEMENTATION AND RESULTS}

\section{A Hardware and software Platform}

Computation of Clustering is based in the simple idea of connecting some computers and using their collective power to solve a problem more quickly. Basically, a cluster consists of a set of computers or processors connected through local network (FDDI, Fast Ethernet) [14] or through the sharing of memory. There are several kinds of cluster [15]; however, the Beowulf cluster has become very popular due to its lower operating cost and its easiness of use [16]. This model is based in PC's (cabinet, mother board, hard disk, memory and NIC) connected through network of computers, using Operating System Open source GNU/Linux and library of communication (MPI/PVM).

The LANE1 cluster used in this work is of Beowulf kind, which is made up of three machines, one server and two slavers machines. The hardware of the cluster is based in Atlhon-Processor $900 \mathrm{MHz}$, with SDRAM memory 256MB , 10 GB Hard disk and is connected through Hub 10/100 Mbits, run by the operating system Linux [17], Red Hat 7.1 distribution, Kernel 2.4, and the library of communication is LAM/MPI [12] version 6.5.3.

At once, the AMAZÔNIA cluster is constituted of eight machines, one master and seven slaves. The master has the following configuration: two Atlhon XP 1800+ processors; DDRAM memory 2 GB; two IDE ATA 133 disks of 60 GB; one RAID ATA 133 controller plate; one Gigabit NIC and one Fast Ethernet NIC. The slaves have the same configuration between them, that is: one Atlhon XP 1800+ processor; $1.5 \mathrm{~GB}$ of DDRAM memory, one IDE ATA 133 disks of $60 \mathrm{~GB}$ and one Fast Ethernet NIC.

The clusters considered here work with free distribution softwares like the Linux operating system (Red Hat 7.3 distribution) and the Message Passing Interface library

\section{$B$ Validation of the obtained results}

The sectorial horn antenna was analysed by using a uniform mesh of $310 \times 248$. The stability of the horn antenna was reached after 800 iterations in the time. In Fig.5, it is shown the distribution of the electric field component $E_{y}$ after 550 iterations in the time. The results presented were validated by comparison with those available in [18].

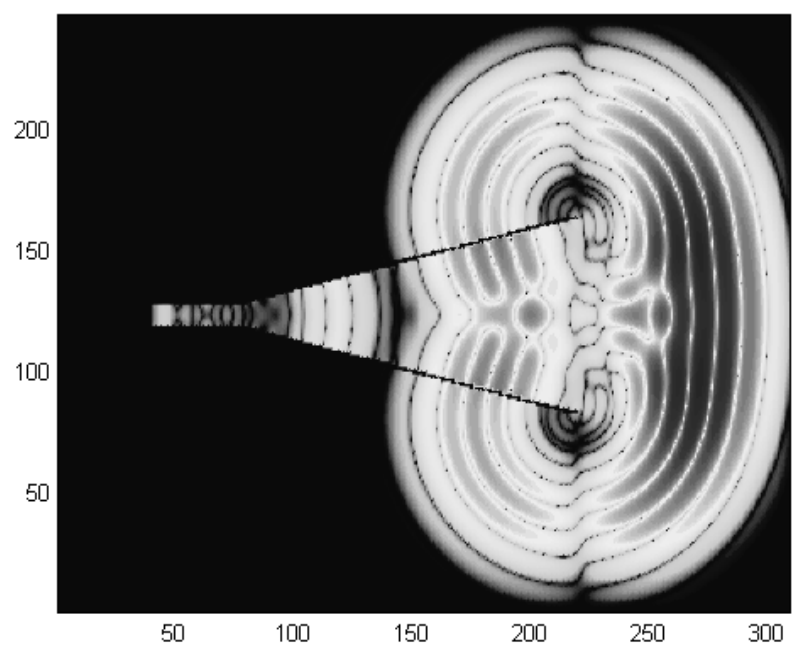

Fig.5. Distribution of the electric field component $E_{y}$ in the region of analysis after 550 iterations in the time. 
In the analysis of cylindrical monopole antenna it was used a two-dimensional mesh of 600x600 and 10000 iterations in the time. For the result shown in Fig.6, it was used $b / a=2.3$, that corresponds to a characteristic impedance of $50 \Omega$ for the coaxial line, $h / a=32.8$ and $\varepsilon_{\mathrm{r}}=1$. The ration of the charactistic time of gaussian pulse $\tau_{p}$ and of charactistic time of the antenna was $\tau_{p} / \tau_{a}=1.61 \times 10^{-1}$. The result shown in Fig.6 corresponds to the voltage computed on B-B' plane (Fig.3). This result has good concordance with the result obtained by Maloney [3], with the difference that in [3] it is calculated the reflected voltage, while in Fig. 6 we have the incident voltage plus the reflected voltage (total voltage). The reflected voltage can be easily obtained by subtracting the total voltage from the incident voltage.

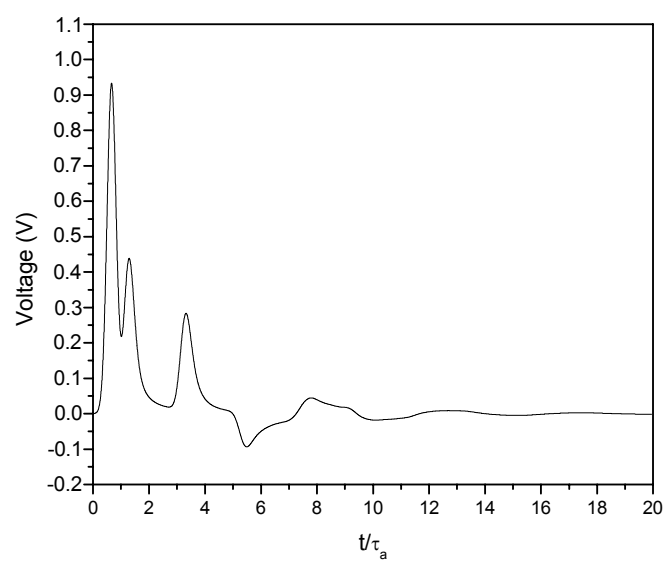

Fig.6. Voltage in the coaxial line for a cylindrical monopole antenna excited by a Gaussian pulse of $1 \mathrm{~V}: \mathrm{b} / \mathrm{a}=2.3, \mathrm{~h} / \mathrm{a}=32.8$, and $\tau_{p} / \tau_{a}=1.61 \times 10^{-1}$.

Figure 7 shows the distribution of the $\mathrm{E}_{\mathrm{z}}$ electric field component in the analysis region after 1200 iteractions in the time for the monopole antenna. In this case the AMAZÔNIALANE02 cluster was used and each square represent the results of each machine.
M5
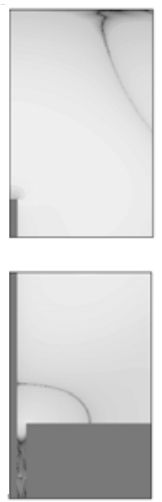

M1
M6
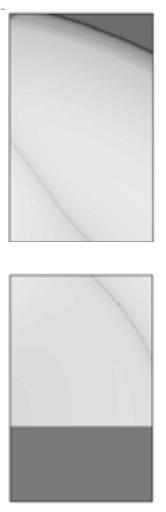

M2
M7
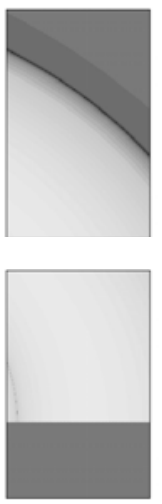

M3
M8
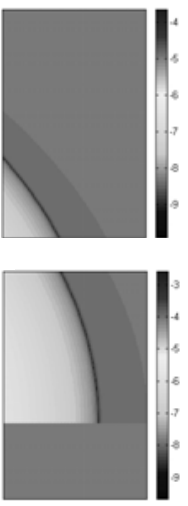

M4
Fig. 7 Distribution of the eletric field component $E_{z}$ in the analysis region after 1200 iteractions in the time.
C Comparison Between the times of serial and parallel processing

To prove the gain of speed obtained with the parallelization of the FDTD code in relation to the sequential, it was made a comparison between the times of processing of the programs executed in both codes.

For the horn antenna, the results of the speedup are shown in Fig. 8 and are related to both the serial execution and for to cluster, with two and three processors.

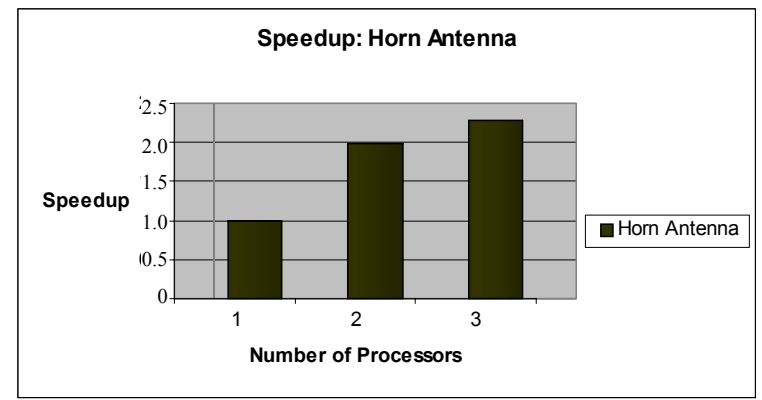

Fig.8. Speedup versus number of processors for the horn antenna.

For the monopole, the results of the speedup are shown in Fig. 9 and are related to both the serial execution and to the cluster, with two, four, six and eight machines.

In this work, the definition of the speedup is $S=T_{S} / T_{N}$, where $T_{S}$ is the time for the sequential processing and $T_{N}$ is the time of the processing for a cluster with $\mathrm{N}$ processors.

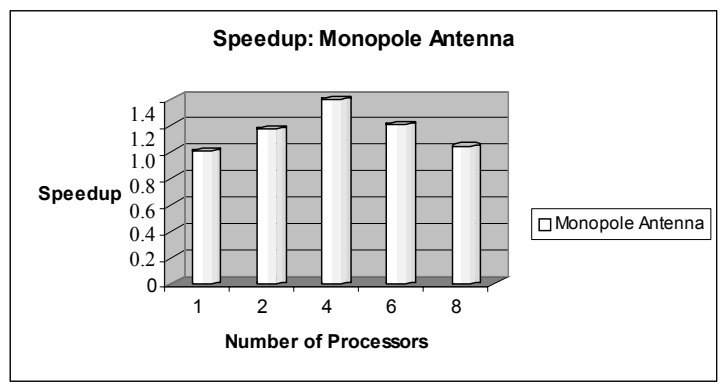

Fig. 9. Speedup versus number of processors for the monopole antenna.

In Fig.10, the time of execution for the horn antenna is represented for both sequential and parallel processing.

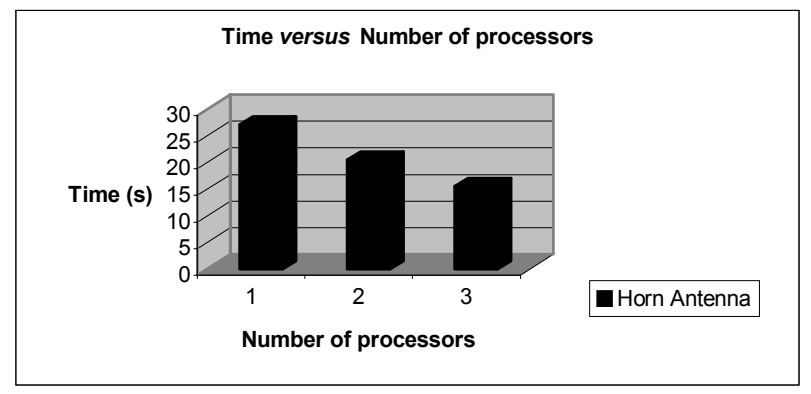

Fig.10. Time of Processing for the Horn Antenna. 
In Fig.11, the time of execution for the monopole antenna is represented for both sequential and parallel processing.

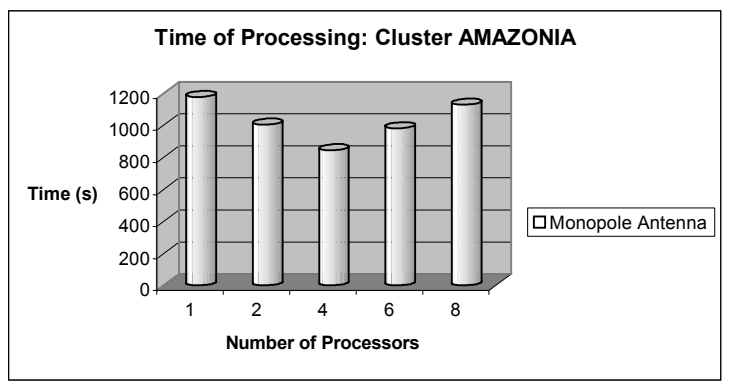

Fig.11 Time of processing for the monopole antenna.

\section{$D$ Analysis and evolution of development}

From Figs. 8 and 9, it is noted that there is a gain of speed, obtained in the parallel implementation, and it is evident that the same has increased with the number of machines and with the size of the problem (time and space discretization) solved. It is worth remembering that in the analysis of the horn antenna, it was used a mesh of $310 \times 248$ and 550 iterations in time; while in the case of the monopole, it was used a mesh of $600 \times 600$ and 10000 iterations.

Apparently, the values presented in Fig. 8 can suggest that the speedup can indefinitely increase only because of the increase of processors. However, the increase of the number of processors and consequently the decrease of the granulation of the problem can take to an excessive increase of communication, Fig. 9, what can take to a process of decrease of the value of the speedup.

\section{CONCLUSIONS}

The FDTD method was applied in the parallel computation of the voltage in the feed line of a monopole antenna and in the analysis of the distribution of field for a E-plane sectorial horn antenna.

The results obtained for each case analysed, both in the parallel and sequential processing, are identical and present an excellent concordance with those available in the literature.

The implementation of the Beowulf cluster (named LANE1 and AMAZÔNIA-LANE02), has shown to be a powerful computational tool that allows the use of the FDTD method in the solution of electromagnetic problems that need great amount of memory and long time of processing.

The parallel computation on an architecture of cluster kind is a strong tendency, for presenting an atractive cost/benefit relation and for the relative easiness of paralleling sequential codes, as for example the FDTD method.

The main goal of this article was to present the relation of processing time redution through the use of parallel computation in the cluster.

\section{REFERENCES}

[1] K.S. Yee, "Numerical solution of initial boundary value problems involving Maxwell's equations in isotropic media,'IEEE Trans. Antennas Propagat., vol. AP-14, pp. 302-307, May 1966.

[2] A. Taflove, "Advances in Computational Electromagnetics - The FiniteDifference Time-Domain Method," Artech House, Boston-London, 1998.

[3] Maloney, J. G. S. Smith, and W. R. Scott, Jr., “ Accurate computation of the radiation from simple antennas using the finite-difference timedomain method," IEEE Trans. Antennas and Proparation, Vol.38, 1990, pp.1059-1068

[4] Berenger, J.P., "A perfectly matched layer for the absorption of electromagnetic waves,"J. Computational Phisics, vol. 114, 1994, pp. 185-200.

[5] J.F.Lee and R.Mittra, "Finite difference time domain algorithm for nonorthogonal grids," Techinal Rep. Electromagnetic Communication Lab., Departament of Eletrical and Computer Engineering, University of Illinois at Urbana-Champaign, 1991.

[6] Almasi, G.S, and Gohlied, A, "Hihgly Parallel Computing". Benjamim/Cummings Publishing, 2aㅡ. ed., 1994.

[7] W.L. Stutzman and G.A. Thiele, "Antenna Theory and Design," $2^{\text {nd }}$ ed., John wiley, New York, 1998.

[8] E. Isaacson and H. B. Keller, "Analysis of Numerical Methods," Wiley, New York, 1967.

[9] S.D. Gedney, "An Anisotropic Perfectely Matched Layer- Absorbing Medium for the Truncation of FDTD Lattices," IEEE Trans. Antennas Propagat., vol. AP-44, No. 12, pp. 1630-1639, Dec. 1996.

[10] G. Mur, "Absorbing boundary conditions for finite-difference approximation of the time-domain electromagnetic field equation," $I E E E$ Trans. Electromagn. Compat., vol. EMC-23, pp. 1073-1077, Nov. 1981.

[11] Foster, I. "Designing and Building Parallel Programs: Concepts and Tools for Parallel Software Engineering, ”. Addison wesley. 1994.

[12] http://www.mpi.nd.edu/lam

[13] Andrews, G. Foundations of Multithreaded, "Parallel and Distributed Programming,", Addison wesley. 2000.

[14] TANENBAUM, ANDREW - "Redes de Computadores," $3^{\circ}$ Ed. Campus - São Paulo, 1996.

[15] http://www.csse.monash.edu.au/ rajkumar/cluster/

[16] http://www.beowulf.org/

[17] http://www.linux.org/

[18] R. O. Santos e C. L. S. S. Sobrinho, “ Análise FDTD - Efeitos de lentes dielétricas na irradiação de antena corneta b-dimensional," IX Simpósio Brasileiro de microondas e Optoeletrônica, pp.608-612, 07-11 August, 2000.

[19] C. A. Balanis, Antenna Theory: Analysis and Design. New York: Wiley, 1997. 